\title{
Neutrophil Activation Probe Imaging Agent
}

National Cancer Institute

\section{Source}

National Cancer Institute. Neutrophil Activation Probe Imaging Agent. NCI Thesaurus.

Code C126277.

A fluorescence imaging agent composed of a fluorescent agent linked, via a human neutrophil elastase (HNE) cleavable peptide, to a dequencher molecule, with imaging activity for diagnostic purposes. Upon local administration, the neutrophil activation probe (NAP) imaging agent, initially quenched, is quickly taken up by activated neutrophils. In turn, the HNE expressed by these cells, specifically cleaves the linker and dequenches the fluorescent agent. The activated fluorescent moiety allows for visualization of activated neutrophils and HNE activity upon using a fluorescence imaging device. Activated neutrophils are upregulated at sites of inflammation and in inflammationinduced cancers, and express high levels of HNE. 